\title{
Stabilization of 2D Assemblies of Silver Nanoparticles by Spin-Coating Polymers
}

\author{
Longyu Hu, Aubrie Pfirman and George Chumanov
}

Department of Chemistry, Clemson University, Clemson 29631 SC 29631, USA

\begin{abstract}
Silver nanoparticles self-assembled on poly(4-vinylpyridine) modified surfaces were spin-coated with poly(methyl methacrylate), poly(butyl methacrylate) and polystyrene from anisole and toluene solutions. The polymers filled the space between the particles thereby providing stabilization of the assemblies against particle aggregation when dried or chemically modified. The polymers did not coat the top surface of the nanoparticles offering the chemical accessibility to the metal surface. This was confirmed by converting the stabilized nanoparticles into silver sulfide and gold clusters. Etching the nanoparticles resulted in crater-like polymeric structures with the cavities extending down to the underlying substrate. Electrochemical reduction of silver inside the craters was performed. The approach can be extended to other nanoparticle assemblies and polymers.
\end{abstract}

Keywords: stabilization; spin-coating; silver nanoparticles; crater-like structures; plasmon resonance

\section{Introduction}

Silver nano particles (Ag NPs) are of great interest due to their unique optical properties stemming from the excitation of plasmon resonances, the collective oscillations of the free electron density.[1] The efficiency of the plasmon resonance excitation in Ag NPs exceeds that of any other mechanism for the interaction of light with matter.[2] The plasmon resonances can be tuned across the near-UV, visible, and near-infrared spectral range by adjusting the particle size, shape, and surrounding dielectric medium. 
When Ag NPs are closely spaced, the coupling between the collective electron oscillations (the plasmon coupling) in individual particles results in new plasmon modes. It was previously demonstrated that the coherent plasmon coupling in 2D arrays of self-assembled Ag NPs produces a sharp resonance that can be used for various sensing applications owing to its sensitivity to the refractive index of the surrounding medium. $[3,4]$ In addition, the enhanced local EM field in spaces between the nanoparticles was utilized for surface enhanced Raman spectroscopy.[5] The plasmon coupling depends on the interparticle distance, particles size and dielectric constant of the surrounding medium. As the dielectric constant increases or the interparticle distance decreases until reaching the optimum value, the coupling becomes stronger for the same size of the particles.[6, 7] However, even a small degree of the surface aggregation has a detrimental effect on the coherent coupling and the sharpness of the resonance.

The self-assembly of nanoparticles is an efficient and easily scalable method for fabricating nanostructured surfaces. It is often challenging to maintain such assemblies without the particle aggregation on the surface, particularly when there is a need for further chemical modifications or the transfer of the assemblies into different media. For example, drying $2 \mathrm{D}$ assemblies of Ag NPs causes the nanoparticles to aggregate into clusters due to the solvent surface tension producing lateral forces on the particles.[8] Consequently, solvents with lower surface tension, from which the self-assembly is carried out, tend to cause less aggregation when drying. It is important to recognize that particles aggregate even when they are chemically attached to the surface that is uniformly modified with affinity ligands as in the case of metal nanoparticles on thiolated surfaces. However, when the affinity ligands are patterned on the surface, the aggregation of nanoparticles is less critical because the particles are physically confined to only patterned areas.[9-11] The aggregation is usually an irreversible process, and the particles remain aggregated after rewetting the assemblies. This causes irreproducibility or complete loss of properties of $\backslash$ nanostructured surfaces.[7]

Malynych et al. stabilized the coupled 2D array of Ag NPs and preserved their sharp plasmon resonance by embedding the arrays into a poly(dimethylsiloxane) matrix. However the particles were covered by the polymer layer rendering the metal surface unsuitable for chemical modifications.[8] Matrubara et al. reported the fabrication of silver nanodisk on both flat and textured surfaces by transfer-printing and the resulted nanostructures were stable toward the aggregation because they were physically anchored to the substrates.[12] In another work, periodic metal nanodot arrays were fabricated by the pulsed laser melting induced fragmentation of prepatterned metal nanostrips.[13] Chichkov et al. reported the use of a laser-induced transfer of molten metal 
nanodroplets for building 2D and 3D arrays.[14] The nanostructures in both reports were stable because the nanoparticles were spatially confined.

The problem of the nanoparticle aggregation in solutions was mainly addressed by chemical modifications of the particles' surface or by encapsulating the particles in protective shells. Negatively charged citrate ions that adsorb on the metal surface stabilize the nanoparticles by the electrostatic repulsion, but such suspensions are limited to low concentrations and the particles aggregate irreversibly upon the addition of electrolytes. $[15,16]$ Thiol groups have high affinity to noble metal surfaces and alkanethiols were used to obtain thermally stable and air-stable metal nanoparticles powders that can be repeatedly redissolved in organic solvent.[17, 18] Amine, phosphine and its oxide, carboxylate, iodine, isocrynide and acetone were also used as stabilizing ligands.[19-26] These small ligands can desorb easily or be replaced by other ligands with stronger affinity to the metal surface.

Nanoparticles coated with a shell of different materials become sterically stabilized and more resistant to high electrolyte concentration. Poly(4-vinylpyridine) (PVP) stabilized Ag NPs are more stable than citrate stabilized Ag NPs in standard media.[27, 28] Polyethylene glycol (PEG) is an inert, amphiphilic and biocompatible linear polymer soluble in water, organic polar and apolar solvents, which forms random coils on the metal nanoparticles' surface when hydrated in water and the particles are stable in electrolytes and biological environments.[29-31] Chitosan is a biodegradable and biocompatible linear polysaccharide used to stabilize noble metal nanoparticles.[32, 33] Dendrimers provide both electrostatic and steric stabilization for nanoparticles, so do polymers that form linear chain structures grafted to the nanoparticles' surface.[34-36] Various silica coated metal nanoparticles were made based on Stöber process usually with controlled thickness by adjusting tetraethyl orthosilicate (TEOS) concentration.[37-40] However, these methods are not very effective for preventing the aggregation of nanoparticles attached to substrates because the particles remain 'free' to roll on the surface. Rubinstein etc. reported metal nanoparticle films on glass stabilized by 3.0-3.5 $\mathrm{nm}$ silica coating that preserved the original plasmon response.[41]

Here, a simple and reliable method for stabilizing 2D assemblies of Ag NPs is reported. The method is based on affixing the nanoparticles to substrates with a PVP layer followed by spin-coating polymers in the space between the nanoparticles. Similar method was previously used to change the dielectric function of the medium to study its effect on the plasmon coupling.[6, 7] By adjusting the spin-coating parameters, the thickness of the polymer layer can be adjusted to stabilize the assemblies without 
coating the metal surface. After etching the nanoparticles, crater-like polymeric structures were obtained. A similar approach was used to produce thin porous silicate films with submicron polystyrene spheres as templates as well as silica films on glassy carbon electrodes with 2D assemblies of hemispherical cavities.[42, 43]

\section{Materials and Methods}

Materials Hydrogen peroxide (30-35\%) from Fisher Science, sulfuric acid (98\%) from J.T.Baker, anisole (>99.0 \%) from Fluka Analytical, ethanol (99.98 \%) from Pharmco-Aaper, silver(I) oxide (99.99\%) powder and Hydrogen tetrachloroaurate (III) (99.99\%) from Alfa Aesar, ferric nitrate nonahydrate (98.7 \%) from Fisher Chemical, poly(4- vinylpyridine) (PVP) [MW 60,000], poly(methylmethacrylate) (PMMA) [MW 996,000], poly(butylmethacrylate) (PBMA) [MW 337,000] and polystyrene (PS) [MW 280,000], sodium sulfide nonahydrate (98\%) from Sigma Aldrich were used as received. Ultrapure water $(<18 \mathrm{M} \Omega . \mathrm{cm})$ was obtained from Millipore-Q system and both ultrahigh purity nitrogen and hydrogen were purchased from Air Gas. Microscope glass slides (VWR) and ITO glass with Rs=8-12 $\Omega /$ sq. (Delta Technologies,) were cut into $25 \times 15 \times 1.0 \mathrm{~mm}$ pieces. $120 \mathrm{~nm}$ thick $\mathrm{Ag}$ and $\mathrm{Au}$ films were deposited via the thermal evaporation in vacuum. All polymer solution concentrations are reported in weight percent.

Instrumentation UV-2501PC Spectrophotometer (Shimadzu) was used to record UV-vis spectra. High-resolution TEM- H9500 (Hitachi) was used to characterize the Ag NPs (Fig. S1). All glass and ITO substrates were cleaned with Plasma Sterilizer PDC-32G (Harrick). AFM measurements were performed in a non-contact mode using AIST-NT SPM Smart system and cantilevers (HQ:NSC14/Al BS-50) from Micromasch. AIST-NT image analysis and processing (Version 3.2.14) software was used for AFM images analysis. Electrochemical Workstation, Model $400 \mathrm{~A}$, (CH Instruments) was used for the electrochemical studies.

Synthesis of Ag NPs The Ag NPs were synthesized by reducing saturated silver oxide solution in ultrapure water by ultrahigh purity hydrogen at $73^{\circ} \mathrm{C}$ in a round bottom flask, as previously reported.[44] The size of the Ag NPs can be adjusted by controlling the reaction time simultaneously monitoring the extinction spectra of the reaction suspension. Colloidal suspensions 
containing $55 \pm 3 \mathrm{~nm}, 100 \pm 5 \mathrm{~nm}$ and $120 \pm 7 \mathrm{~nm}$ Ag NPs were used in the study.

2D assemblies of Ag NPs 25x15x1.0 mm glass substrates were treated by a mixture of 35\% hydrogen peroxide and $98 \%$ sulphuric acid for 4 hours, washed with copious amount DI water, dried with pure nitrogen and cleaned with plasma. Clean substrates were rolled on a hot-dog style roller in $0.01 \%$ PVP ethanol solution for 4.5 hours, and rinsed several times with ethanol followed by the final rinse with DI water. The PVP modified substrates were exposed at constant agitation to Ag NP suspensions $(\mathrm{OD}=0.1,0.3)$ for different times ranging from 2.5 to13 hours followed by rinsing with water to obtain $2 \mathrm{D}$ assemblies with different nanoparticle density.

Polymer spin-coating and etching of Ag NPs The 2D assemblies of Ag NPs were immersed first in pure anisole for a few seconds to remove the excess of surface water followed by immersing into polymer anisole solutions (0.6 \% PMMA, $1 \%$ PMMA, $1.2 \%$ PMMA, $1.8 \%$ PMMA, $3 \%$ PMMA; $1 \%$ PBMA; $1 \%$ PS, $2 \%$ PS, $3 \%$ PS) for 2 minutes. The wet substrates were mounted on the spin coater and additional $30 \mu \mathrm{L}$ of the corresponding polymer solution was dropped onto the substrates followed by spinning at $5000 \mathrm{rpm}$ for 45 seconds. The substrates were annealed at $140^{\circ} \mathrm{C}$ for 10 minutes. Etching the Ag NPs, was performed by dropping $45 \mu \mathrm{L}$ of $1.5 \mathrm{M} \mathrm{Fe}\left(\mathrm{NO}_{3}\right)_{3}$ aqueous solution onto the substrates followed by through rinsing with DI water after a few seconds of the exposure. The procedures are schematically presented in Fig. 1.

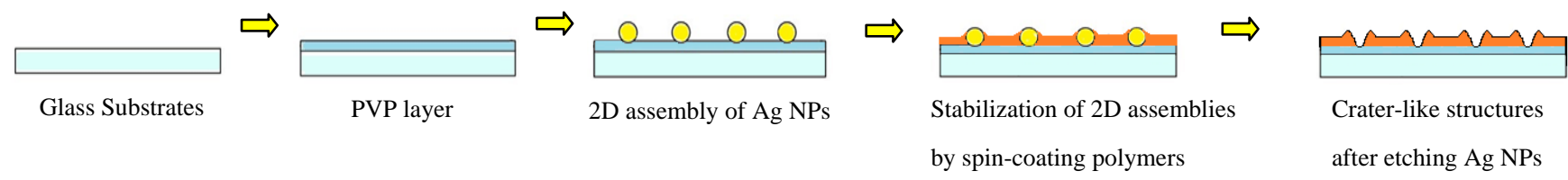

Fig. 1. Stabilization of $2 \mathrm{D}$ assemblies and formation of crater-like structures

Electrochemical silver deposition into craters PMMA-based craters were formed on ITO, Au or Ag coated substrates. Mixture of $0.1 \mathrm{M} \mathrm{Na}_{2} \mathrm{SO}_{4}$ and $0.1 \mathrm{mM} \mathrm{Ag}_{2} \mathrm{SO}_{4}$ deoxygenated by bubbling nitrogen gas for at least 15 minutes was used as electrolyte in all electrochemical experiments. The metal deposition was carried out in the 'bulk electrolysis with coulometry' mode at potentials between $-0.57 \mathrm{~V}$ and $-0.75 \mathrm{~V}$ vs. SHE for 3-10 seconds depending upon the desired metal thickness. 


\section{Results and Discussion}

Unmodified microscope substrates made of float glass have an average roughness less than $5 \mathrm{~nm}$. The average roughness was uniformly increased to about 8-9 $\mathrm{nm}$ after the PVP modification carried out from $0.01 \%$ ethanol solution for 4 hours. The roughness was further increased when higher PVP concentrations were used. It is well recognized that high polymer concentrations favor more coiled structures of polymer molecules on surfaces leading to larger roughness.[45] The exposure of the modified substrates to aqueous $100 \mathrm{~nm}$ Ag NPs suspensions resulted in the 2D self-assembly of Ag NPs. The density of the particles on the surface was controlled by adjusting the particle concentration in the suspension as well as the exposure time. The self-assembly was carried out from DI water with low ionic strength providing long range electrostatic repulsion between the particles due to the electric double layer associated with the metal surface. The electrostatic repulsion kept the individual particles well separated in the assembly. The Ag NPs absorb on the PVP surface through the formation of bonds between the lone pair electrons on the pyridyl ring and the silver surface. Despite this strong interaction, the particles can move on the surface. In order to move a particle to another location, the bond between the particle and the surface needs to be broken; however a new identical bond is formed at the new location. This movement can be viewed as breaking of existing and simultaneous forming new surface bonds, the process that does not require additional energy. The particle assemblies in water are stabilized by the electrostatic repulsion, but drying negates this repulsion as well as produces lateral forces that push the particle together resulting in the aggregation. The lateral forces are caused by the surface tension of drying water. In this work, the stabilization of the particles in the assemblies was achieved by filling the spaces between the particles with polymers. The stabilized assembly could withstand repeated drying/rewetting cycles as well as various chemical modifications while retaining their original arrangement. The method can be applied to other polymer/nanoparticle assembly combinations.

The average height of Ag NPs in the assemblies, as measured with the AFM, was $97 \pm 4 \mathrm{~nm}$ (Fig. 2a). The particles appeared with sharp corners suggesting that their surface was not covered with the PVP (Fig. S2). It was initially suspected that, because of the high affinity of PVP to silver, the polymer molecules adsorbed on the surface of the substrate can also partially cover the particle surface to establish an equilibrium distribution between the two surfaces. 

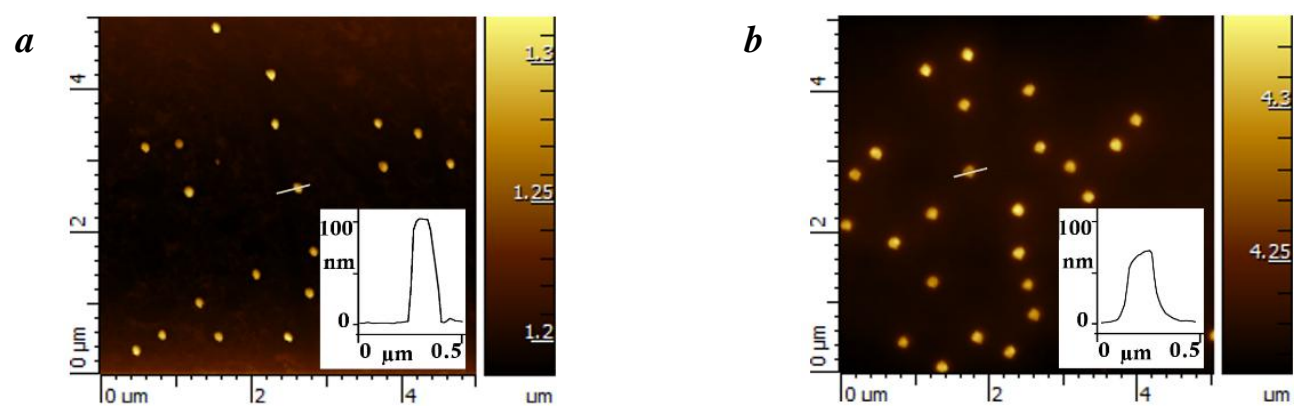

Fig. 2. AFM images of $100 \mathrm{~nm}$ Ag NPs self-assembled on PVP coated glass substrates: (a) before polymer spin- coating; (b) after spin-coating PS from anisole solution. Insets: height profiles of Ag NPs

To prevent the particle aggregation, the $2 \mathrm{D}$ assemblies were stabilized by spin-coating various concentrations of polymers such as PS, PMMA, PBMA from two different solvents, toluene and anisole. After the spin-coating, the average height of the particles decreased depending upon the concentration of the polymer, a general trend observed for all polymers and solvents used in this work. For example, when the PS concentration was increased from $0.3 \%$ to $1 \%$ and $3 \%$, the average height of Ag NPs decreased from $97 \pm 3 \mathrm{~nm}$ to $90 \pm 3 \mathrm{~nm}$ and $16 \pm 3 \mathrm{~nm}$ respectively. Further height decrease on the order of a few nm was observed after the substrates coated with $1 \%$ PS or $1 \%$ PMMA were annealed at $150^{\circ} \mathrm{C}$ for 25 minutes. The measured decrease of the particles' height after the annealing seems counterintuitive. One would expect an increase of the measured height because the annealing at temperatures larger than that of the glass transition $\left(\sim 110^{\circ} \mathrm{C}\right)$ of PS and PMMA will cause the relaxation of the polymers causing the particles to float to the surface of the polymer layer as neither PS nor PMMA have strong affinity to silver. The relaxation of the polymer layer was concluded from the AFM measurements of the surface roughness that changed from 4-5 $\mathrm{nm}$ to $1-2 \mathrm{~nm}$ for $1 \%$ polystyrene film and from $7-8 \mathrm{~nm}$ to $1-2 \mathrm{~nm}$ for $1 \%$ PMMA film. The discrepancy can be explained by considering the underlying PVP layer that will also soften at this temperature and pull the particles deeper inside the layers due to the PVPs strong affinity to silver. Mixing of PVP layer with PS or PMMA layers is also possible at this temperature.

When the 2D assemblies of Ag NPs are stabilized by the spin-coating of polymers, it is important to know whether or not the metal surface is coated with the polymer. To address this question, etching of nanoparticles as well as chemical modifications of the metal surface was undertaken. Ferric nitrate can etch silver because its standard reduction potential $(0.77 \mathrm{~V})$ is close to that of 
$\mathrm{Ag}^{+}(0.80 \mathrm{~V})$ and the resultant silver nitrate is a soluble compound.[46] After the etching of the assemblies with molar excess of

ferric nitrate crater-like structures were observed thereby indicating that the silver surface was not covered with the polymers or, at least, the polymer layers were not sufficiently dense to prevent chemical accessibility to the metal surface (Fig. 2b and Fig. 3). The height and the external diameter of the craters, as measured relative the area of the substrate containing no nanoparticles, depended on both the type and the concentration of the polymer. For example, $100 \mathrm{~nm}$ Ag NPs and $1 \%$ of PBMA, PS and PMMA yielded the average height of $40 \pm 3 \mathrm{~nm}, 52 \pm 3 \mathrm{~nm}$, and $50 \pm 4 \mathrm{~nm}$ whereas external diameters were $440-550 \mathrm{~nm}$, and $180-310 \mathrm{~nm}$, 280-350 nm, respectively. No significant differences of the crater structures were found for glass and ITO substrates under the same conditions (Fig. S3). Although the crater depth measurements were not reliable due to the limited penetration of the AFM tip inside the craters, there was a noticeable trend in that higher concentrations of polymers resulted in deeper craters. The solvent, in which the polymers were dissolved prior to spin-coating, had also effect on the crater dimensions, e.g. $1 \%$ PS in anisole consistently gave taller craters than $1 \%$ PS in toluene.[47] Proportionally smaller craters were observed when smaller Ag NPs were used as templates.

Whereas the dependence of crater dimensions upon the concentration of polymers is rather clear, the dependence upon the type of the polymer, substrate and solvent most likely reflected how the polymer solutions wetted both the substrate and the nanoparticles. The underlying layer of PVP on the substrate is expected to be wetted well by both solvents because of $\pi-\pi$ interactions between the aromatic rings. Neither of the polymers nor solvents is expected to have specific affinity to the silver surface, so it is likely that all solutions wetted the surface of the nanoparticles to the same degree. The formation of the craters was due to the capillary force that pulled up the polymer solutions at the particle substrate interface. In the absence of strong interactions between a surface and a liquid, the capillary force depends upon the ratio of the surface tension to solvent density and is larger for anisole polymer solutions thereby leading to consistently larger craters formed from anisole than those from toluene. The dissolved polymers did not change substantially the surface tension from that of the pure solvents as was tested using the drop size method for $1 \%$ polymer solutions. In this method, drops of the same volume of different polymers solutions were deposited on clean glass substrates and the spot sizes were compared to that of pure solvents. No statistically meaningful differences of the spot sizes were noticed thereby concluding that the polymers at such low concentration did not affect the surface tension of the 
solvents.
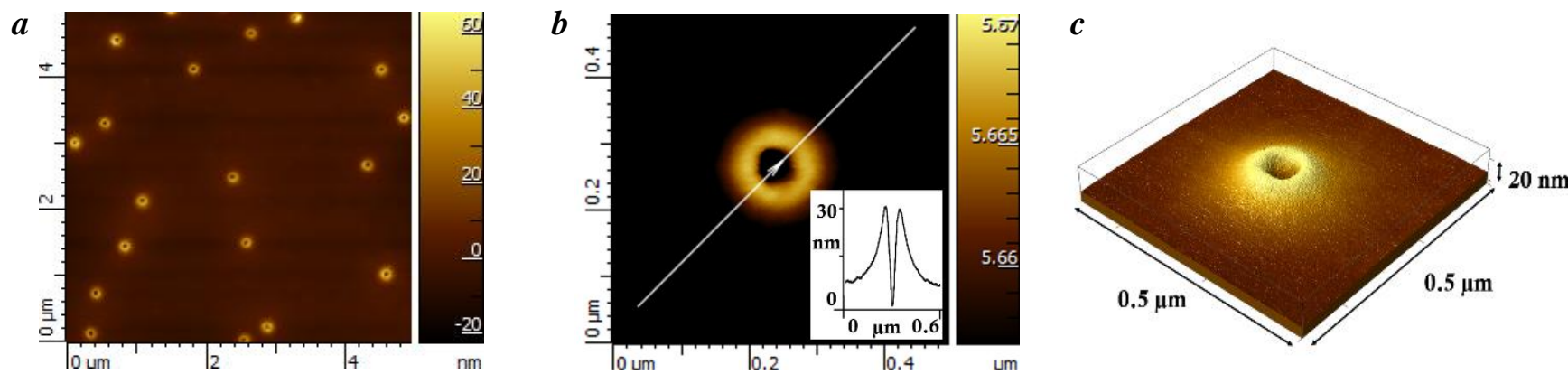

Fig. 3. AFM images of crater-like structures after etching $\mathrm{Ag} \mathrm{NPs}$ with $\mathrm{Fe}\left(\mathrm{NO}_{3}\right)_{3}$. Inset: the height profile of a crater

Polymer stabilized 2D assemblies of $\mathrm{Ag}$ NPs were exposed to $\mathrm{HAuCl}_{4}$ aqueous solution with the expectation that the gold

salt will etch silver away producing gold nanoparticle assemblies. Indeed, the AFM imaging revealed the presence of nearly

spherical particle clusters protruding on average $119 \pm 5 \mathrm{~nm}$ from the surface of the substrates and arranged in the same way as the original Ag NPs, Fig. 4a. Each cluster was composed of roughly 5 smaller gold particles with the estimated size of $30 \mathrm{~nm}$. The $\mathrm{UV}$-Vis spectroscopy confirmed the presence of the plasmon resonance peak at about $547 \mathrm{~nm}$ corresponding to small Au NPs as well as a weak shoulder at $645 \mathrm{~nm}$ due to the plasmon coupling between the particles in the clusters (Fig. 4b). It was concluded that each Ag NP was completely dissolved because the strong plasmonic features from the Ag NPs disappeared from the UV-Vis spectra after etching with the gold salt.

The structure of the gold clusters depended upon the surface density of Ag NPs on the substrate. The size of the gold clusters was smaller and they appeared more like open circles on the rim of the crates as the Ag NPs density decreased (Fig. 4c). This observation was rationalized as interplay between the local concentration of the zero valence gold and its diffusion from the substrate. Low surface densities of Ag NPs lead to low local concentrations of the zero valance gold near the substrate. This zero valence gold partially diffused away from the substrate before it had a chance to assemble into small nanoparticles that became attached to the rim of the craters. At high silver densities, more zero valance gold was available near craters producing more Au NPs that formed 3D gold clusters on top of the craters. The zero valance gold that diffused from the substrate most likely formed Au NPs in the solution; however it was impossible to detect them using UV-Vis spectroscopy to due to their low concentration. 

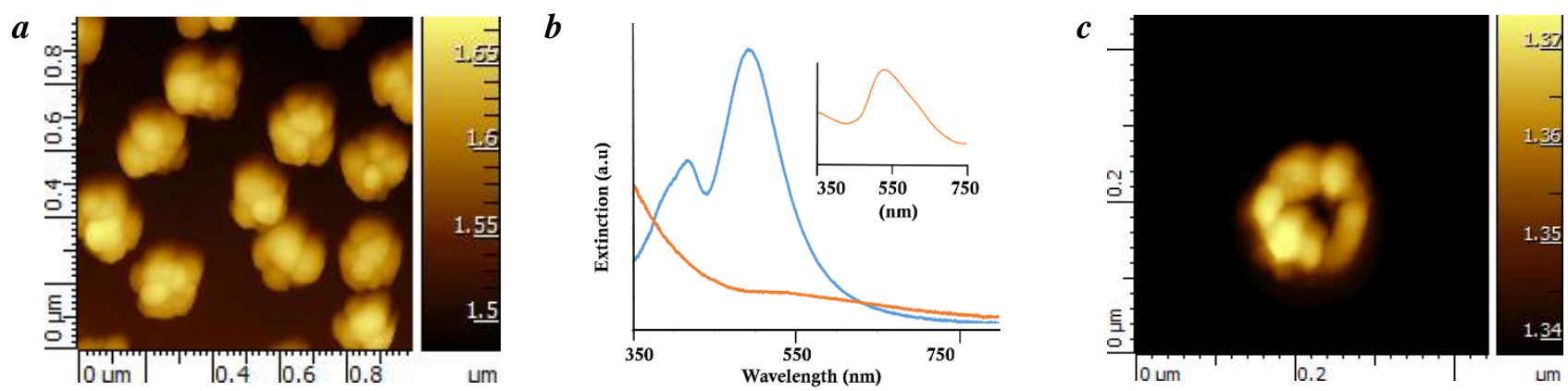

Fig. 4. AFM images (a) and (c) of $2 \mathrm{D}$ assemblies of Au clusters obtained by $\mathrm{HAuCl}_{4}$ treatment of $2 \mathrm{D}$ assemblies of $120 \mathrm{~nm} \mathrm{Ag}$ NPs stabilized by spin-coating $1 \%$

PMMA; (b) UV-Vis spectra of 2D assembly before (blue curve) and after (orange curves) the treatment. Inset: zoom-in UV-Vis spectrum after the treatment.

The polymer stabilized 2D assemblies of $\mathrm{Ag}$ NPs was also treated with $\mathrm{Na}_{2} \mathrm{~S}$ aqueous solutions. AFM images revealed the simultaneous presence of small nanoparticle clusters and empty craters on the substrates (Fig. 5a). The average measured height of the clusters was $94 \pm 5 \mathrm{~nm}$. The plasmon resonance broadened and shifted to the red spectral region due to the formation of silver sulfide on the surface of the NPs (Fig. 5b and Fig.5c). Silver sulfide has a large dielectric function and absorption coefficient casing the red shift and dampening of the resonance. The formation of silver sulfide on Ag NPs appeared to be a self-limiting reaction as large changes in the UV-Vis spectra were observed in the first minutes after the exposure to $\mathrm{Na}_{2} \mathrm{~S}$ followed by only small changes with time. As expected, the rate of the silver sulfide formation in the case of 2D assemblies without spin-coating polymer layer was slightly larger (Fig. 5c). The fact that the $2 \mathrm{D}$ assemblies of $\mathrm{Ag}$ NPs treated with $\mathrm{Na}_{2} \mathrm{~S}$ retained their plasmonic spectral features, albeit dampened, suggested that the nanoparticles composed of a silver core coated with small silver sulfide particles, as was evident from close-up AFM images of individual nanoparticles (Fig. 5a, Inset). The empty craters that were also observed most likely appeared after rinsing the substrates exposed to $\mathrm{Na}_{2} \mathrm{~S}$ with water. Silver sulfide coated nanoparticles are expected to have no/low affinity to PVP modified substrates and can be easily washed away.
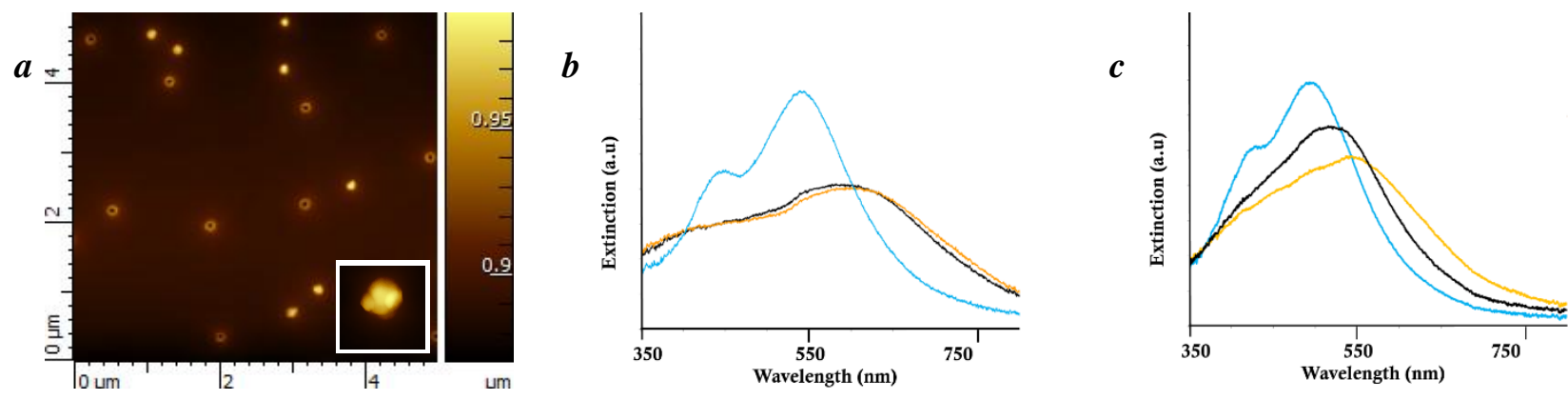
Fig. 5. (a) AFM image of $2 \mathrm{D}$ assembly of Ag NPs stabilized by spic-coating of $1 \% \mathrm{PS}$ after treatment with $0.16 \mathrm{M} \mathrm{Na} 2 \mathrm{~S}$ for 3 min. Inset: close-up image of one particle. UV-Vis spectra of the stabilized (b) and unstabilized (c) assemblies before (blue curve) and after the treatment with $0.11 \mathrm{M}$ Na2S for 2 min (black curve) and 20 min (orange curve).

Attempts were also made to electrochemically grow metallic silver in the craters. ITO, vacuum deposited gold or silver conducting substrates were coated with PVP followed by attaching Ag NPs, spin-coating of PMMA and etching the nanoparticles to produce craters. The substrates containing craters were connected to the electrochemical workstation and the silver reduction was carried out at $-0.58 \mathrm{~V}$ and $-0.71 \mathrm{~V}$ vs. SHE. The growth of silver was not uniform on all three substrates with the gold substrates exhibited the most consistent results (Fig. S4). AFM imaging revealed craters filled with silver together with empty craters as well as the presence of various size clusters of small Ag NPs in the vicinity of the craters (Fig. 6). The nonuniform electrochemical growth of silver was most likely due to the heterogeneity of the underlying conducting substrates. It is well known, for example, that the electrochemical deposition of metals on bare ITO proceeds via seeded growth, in which the metal reduction begins at defect sites, often at the boundaries between individual ITO crystallites that provide the smallest reduction overpotential.[48] Silver reduction occurred in places where a crater overlapped with such defect site. In some instances, zero silver diffused from the inside of craters forming silver clusters on the outside. More negative reduction potentials resulted in the formation of larger silver particles/clusters and decreased the number of empty craters.
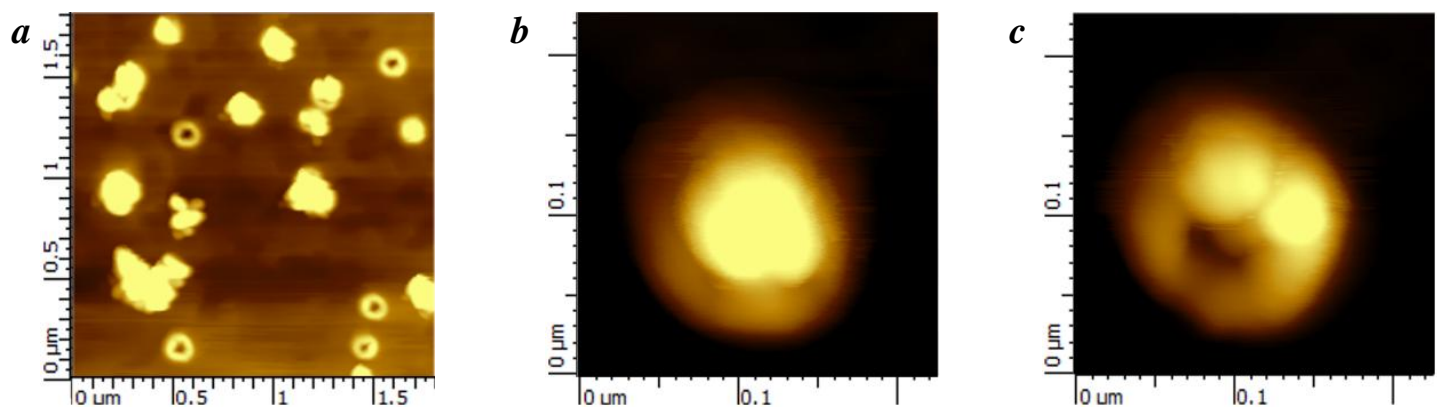

Fig. 6. (a) (b) (c) AFM images of electrochemically reduced silver onto crater-like structures produced on a ITO substrate by etching $2 \mathrm{D}$ assembly of 100 nm Ag NPs stabilized by spin-coating $1.2 \%$ PMMA. Reduction conditions: $-0.75 \mathrm{~V}$ vs. SHE, 3 seconds.

\section{Conclusions}

Spin-coating of polymers onto 2D assemblies of Ag NPs was used to stabilize the assemblies against aggregation. The polymer 
filled the space between the particles leaving the metal surface uncoated and accessible to various chemical reactions. Etching

nanoparticles produced crater-like structures, the size of which can be manipulated by changing the polymer concentration and the solvent. The method can be extended to other polymers and nanoparticle assemblies.

\section{Corresponding author}

George Chumanov

*E-mail: gchumak@clemson.edu

Phone: (864) 656-2339

Fax: (864) 656-0567

Department of Chemistry, Clemson University, Clemson City, SC 29631, USA

\section{Acknowledgments}

This research was supported by Department of Energy, Grant DE-FG02-06ER46342. The authors also thank Daniel Willett for assistance with obtaining $100 \mathrm{~nm} \mathrm{Ag} \mathrm{NPs.}$

\section{References}

[1] U. Kreibig, M. Vollmer, Optical Properties of Metal Clusters, Springer, Berlin 1995.

[2] D.D. Evanoff, G. Chumanov, Size-controlled synthesis of nanoparticles. 2. Measurement of extinction, scattering, and absorption cross sections, Journal of Physical Chemistry B, 108 (2004) 13957-13962. doi: 10.1021/jp0475640.

[3] J.K. Daniels, G. Chumanov, Nanoparticle-mirror sandwich substrates for surface-enhanced Raman scattering, Journal of Physical Chemistry B, 109 (2005) 17936-17942. doi: 10.1021/jp053432a.

[4] S.D. Hudson, G. Chumanov, Bioanalytical applications of SERS (surface-enhanced Raman spectroscopy), Anal. Bioanal. Chem., 394 (2009) 679-686. doi: 10.1007/s00216-009-2756-2.

[5] M.P. Konrad, A.P. Doherty, S.E.J. Bell, Stable and Uniform SERS Signals from Self-Assembled Two-Dimensional Interfacial Arrays of Optically Coupled Ag Nanoparticles, Analytical Chemistry, 85 (2013) 6783-6789. doi: 10.1021/ac4008607.

[6] M.K. Kinnan, G. Chumanov, Plasmon Coupling in Two-Dimensional Arrays of Silver Nanoparticles: II. Effect of the Particle Size and Interparticle Distance, Journal of Physical Chemistry C, 114 (2010) 7496-7501. doi: 10.1021/jp911411x.

[7] M.K. Kinnan, S. Kachan, C.K. Simmons, G. Chumanov, Plasmon Coupling in Two-Dimensional Arrays of Silver Nanoparticles: I. Effect of the Dielectric Medium, Journal of Physical Chemistry C, 113 (2009) 7079-7084. doi:

10.1021/jp900090a.

[8] S. Malynych, H. Robuck, G. Chumanov, Fabrication of two-dimensional assemblies of Ag nanoparticles and nanocavities in poly(dimethylsiloxane) resin, Nano Letters, 1 (2001) 647-649. doi: 10.1021/n1015604v.

[9] S.J. Barcelo, S.-T. Lam, G.A. Gibson, X. Sheng, D. Henze, Nanosphere lithography based technique for fabrication of large area, well ordered metal particle arrays, Alternative Lithographic Technologies Iv, 8323 (2012). doi: 832321 
10.1117/12.916600.

[10] J.D. Le, Y. Pinto, N.C. Seeman, K. Musier-Forsyth, T.A. Taton, R.A. Kiehl, DNA-templated self-assembly of metallic nanocomponent arrays on a surface, Nano Letters, 4 (2004) 2343-2347. doi: 10.1021/n1048635+.

[11] J. Zheng, P.E. Constantinou, C. Micheel, A.P. Alivisatos, R.A. Kiehl, N.C. Seeman, Two-dimensional nanoparticle arrays show the organizational power of robust DNA motifs, Nano Letters, 6 (2006) 1502-1504. doi: 10.1021/n1060994c.

[12] H. Mizuno, T. Kaneko, I. Sakata, K. Matsubara, Capturing by self-assembled block copolymer thin films: transfer printing of metal nanostructures on textured surfaces, Chemical Communications, 50 (2014) 362-364. doi: 10.1039/c3cc46198j.

[13] Q. Xia, S.Y. Chou, The fabrication of periodic metal nanodot arrays through pulsed laser melting induced fragmentation of metal nanogratings, Nanotechnology, 20 (2009). doi: 285310

10.1088/0957-4484/20/28/285310.

[14] A.I. Kuznetsov, R. Kiyan, B.N. Chichkov, Laser fabrication of 2D and 3D metal nanoparticle structures and arrays, Optics Express, 18 (2010) 21198-21203. doi: 10.1364/oe.18.021198.

[15] R.A. Sperling, W.J. Parak, Surface modification, functionalization and bioconjugation of colloidal inorganic nanoparticles,

Philos. Trans. R. Soc. A-Math. Phys. Eng. Sci., 368 (2010) 1333-1383. doi: 10.1098/rsta.2009.0273.

[16] T. Yonezawa, T. Kunitake, Practical preparation of anionic mercapto ligand-stabilized gold nanoparticles and their immobilization, Colloids and Surfaces a-Physicochemical and Engineering Aspects, 149 (1999) 193-199. doi:

10.1016/s0927-7757(98)00309-4.

[17] M. Brust, M. Walker, D. Bethell, D.J. Schiffrin, R. Whyman, Synthesis of thiol-derivatised gold nanoparticles in a two-phase Liquid-Liquid system, Journal of the Chemical Society-Chemical Communications, (1994) 801-802. doi: 10.1039/c39940000801. [18] J.C. Love, L.A. Estroff, J.K. Kriebel, R.G. Nuzzo, G.M. Whitesides, Self-assembled monolayers of thiolates on metals as a form of nanotechnology, Chemical Reviews, 105 (2005) 1103-1169. doi: 10.1021/cr0300789.

[19] W.L. Cheng, S.J. Dong, E.K. Wang, Iodine-induced gold-nanoparticle fusion/fragmentation/aggregation and iodine-linked nanostructured assemblies on a glass substrate, Angewandte Chemie-International Edition, 42 (2003) 449-+. doi: 10.1002/anie.200390136.

[20] M.C. Daniel, D. Astruc, Gold nanoparticles: Assembly, supramolecular chemistry, quantum-size-related properties, and applications toward biology, catalysis, and nanotechnology, Chemical Reviews, 104 (2004) 293-346. doi: 10.1021/cr030698+.

[21] H.S. Kim, S.J. Lee, N.H. Kim, J.K. Yoon, H.K. Park, K. Kim, Adsorption characteristics of 1,4-phenylene diisocyanide on gold nanoparticles: Infrared and Raman spectroscopy study, Langmuir, 19 (2003) 6701-6710. doi: 10.1021/la026756o.

[22] D.V. Leff, L. Brandt, J.R. Heath, Synthesis and characterization of hydrophobic, organically-soluble gold nanocrystals functionalized with primary amines, Langmuir, 12 (1996) 4723-4730. doi: 10.1021/la960445u.

[23] G.T. Li, M. Lauer, A. Schulz, C. Boettcher, F.T. Li, J.H. Fuhrhop, Spherical and planar gold(0) nanoparticles with a rigid gold(I)-anion or a fluid gold (0)-acetone surface, Langmuir, 19 (2003) 6483-6491. doi: 10.1021/la0300277.

[24] P.R. Selvakannan, S. Mandal, S. Phadtare, R. Pasricha, M. Sastry, Capping of gold nanoparticles by the amino acid lysine renders them water-dispersible, Langmuir, 19 (2003) 3545-3549. doi: 10.1021/la026906v.

[25] W.W. Weare, S.M. Reed, M.G. Warner, J.E. Hutchison, Improved synthesis of small (d(CORE) approximate to $1.5 \mathrm{~nm})$ phosphine-stabilized gold nanoparticles, Journal of the American Chemical Society, 122 (2000) 12890-12891. doi: 10.1021/ja002673n.

[26] M. Yamamoto, M. Nakamoto, New type of monodispersed gold nanoparticles capped by myristate and $\mathrm{PPh} 3$ ligands prepared by controlled thermolysis of $\mathrm{Au}(\mathrm{C} 13 \mathrm{H} 27 \mathrm{COO})(\mathrm{PPh} 3)$, Chemistry Letters, 32 (2003) 452-453. doi: 10.1246/cl.2003.452.

[27] T. Sakura, T. Takahashi, K. Kataoka, Y. Nagasaki, One-pot preparation of mono-dispersed and physiologically stabilized gold colloid, Colloid and Polymer Science, 284 (2005) 97-101. doi: 10.1007/s00396-005-1339-9.

[28] M. Tejamaya, I. Roemer, R.C. Merrifield, J.R. Lead, Stability of Citrate, PVP, and PEG Coated Silver Nanoparticles in Ecotoxicology Media, Environmental Science \& Technology, 46 (2012) 7011-7017. doi: 10.1021/es2038596.

[29] B. Ballou, B.C. Lagerholm, L.A. Ernst, M.P. Bruchez, A.S. Waggoner, Noninvasive imaging of quantum dots in mice, Bioconjugate Chem., 15 (2004) 79-86. doi: 10.1021/bc034153y.

[30] T.J. Daou, L. Li, P. Reiss, V. Josserand, I. Texier, Effect of Poly(ethylene glycol) Length on the in Vivo Behavior of Coated Quantum Dots, Langmuir, 25 (2009) 3040-3044. doi: 10.1021/la8035083.

[31] R. Gref, M. Luck, P. Quellec, M. Marchand, E. Dellacherie, S. Harnisch, T. Blunk, R.H. Muller, 'Stealth' corona-core 
nanoparticles surface modified by polyethylene glycol (PEG): influences of the corona (PEG chain length and surface density) and of the core composition on phagocytic uptake and plasma protein adsorption, Colloids and Surfaces B-Biointerfaces, 18 (2000) 301-313. doi: 10.1016/s0927-7765(99)00156-3.

[32] K. Esumi, N. Takei, T. Yoshimura, Antioxidant-potentiality of gold-chitosan nanocomposites, Colloids and Surfaces B-Biointerfaces, 32 (2003) 117-123. doi: 10.1016/s0927-7765(03)00151-6.

[33] H.Z. Huang, X.R. Yang, Synthesis of chitosan-stabilized gold nanoparticles in the absence/presence of tripolyphosphate, Biomacromolecules, 5 (2004) 2340-2346. doi: 10.1021/bm0497116.

[34] G. Fritz, V. Schadler, N. Willenbacher, N.J. Wagner, Electrosteric stabilization of colloidal dispersions, Langmuir, 18 (2002) 6381-6390. doi: 10.1021/la015734j.

[35] Y.A. Wang, J.J. Li, H.Y. Chen, X.G. Peng, Stabilization of inorganic nanocrystals by organic dendrons, Journal of the American Chemical Society, 124 (2002) 2293-2298. doi: 10.1021/ja016711u.

[36] J. Zheng, M.S. Stevenson, R.S. Hikida, P.G. Van Patten, Influence of pH on dendrimer-protected nanoparticles, Journal of Physical Chemistry B, 106 (2002) 1252-1255. doi: 10.1021/jp013108p.

[37] K. Chen, C.-C. Lin, J. Vela, N. Fang, Multishell Au/Ag/SiO2 Nanorods with Tunable Optical Properties as Single Particle Orientation and Rotational Tracking Probes, Analytical Chemistry, 87 (2015) 4096-4099. doi: 10.1021/acs.analchem.5b00604.

[38] S. Raj, P. Rai, S.M. Majhi, Y.-T. Yu, Morphology controlled Ag@SiO2 core-shell nanoparticles by ascorbic acid reduction, Journal of Materials Science-Materials in Electronics, 25 (2014) 1156-1161. doi: 10.1007/s10854-013-1702-4.

[39] W. Stober, A. Fink, E. Bohn, Controlled growth of monodisperse silica spheres in the micron size range, Journal of Colloid and Interface Science, 26 (1968) 62-69. doi: 10.1016/0021-9797(68)90272-5.

[40] N.M. Khaa, C.-H. Chen, W.-N. Sub, J. Rickb, B.-J. Hwang, Improved Raman and photoluminescence sensitivity achieved using bifunctional Ag@SiO2 nanocubes, Phys. Chem. Chem. Phys., (2015). doi: 10.1039/C4CP05217J.

[41] Y. Chaikin, O. Kedem, J. Raz, A. Vaskevich, I. Rubinstein, Stabilization of Metal Nanoparticle Films on Glass Surfaces Using Ultrathin Silica Coating, Analytical Chemistry, 85 (2013) 10022-10027. doi: 10.1021/ac402020u.

[42] M. Kanungo, P.N. Deepa, M.M. Collinson, Template-directed formation of hemispherical cavities of varying depth and diameter in a silicate matrix prepared by the sol-gel process, Chemistry of Materials, 16 (2004) 5535-5541. doi: 10.1021/cm049270x.

[43] A.N. Khramov, J. Munos, M.M. Collinson, Preparation and characterization of macroporous silicate films, Langmuir, 17 (2001) 8112-8117. doi: 10.1021/la010112j.

[44] D.D. Evanoff, G. Chumanov, Size-controlled synthesis of nanoparticles. 1. "Silver-only" aqueous suspensions via hydrogen reduction, Journal of Physical Chemistry B, 108 (2004) 13948-13956. doi: 10.1021/jp047565s.

[45] S. Malynych, I. Luzinov, G. Chumanov, Poly(vinyl pyridine) as a universal surface modifier for immobilization of nanoparticles, Journal of Physical Chemistry B, 106 (2002) 1280-1285. doi: 10.1021/jp013236d.

[46] Y.N. Xia, E. Kim, G.M. Whitesides, Microcontact printing of alkanethiols on silver and its application in microfabrication, J. Electrochem. Soc., 143 (1996) 1070-1079. doi: 10.1149/1.1836585.

[47] A.L. Pfirman, The Fabrication And Study Of Silver Nanoparticle Structures, Chemistry, Clemson University, 2013.

[48] G.B. Gonzalez, Investigating the Defect Structures in Transparent Conducting Oxides Using X-ray and Neutron Scattering Techniques, Materials, 5 (2012) 818-850. doi: 10.3390/ma5050818. 